\title{
Atomic Spectroscopy N \\ In situ Investigation of the Valence States of Iron-bearing Phases in Chang'E-5 Lunar Soil using FIB, AES, and TEM-EELS Techniques
}

\author{
Bing Mo, ${ }^{\text {a,b,c,d }}$ Zhuang Guo, ${ }^{\text {a,d }}$ Yang Li, ${ }^{\text {ac, }, * *}$ Dan Zhu, ${ }^{\text {b,c }}$ Xiaojia Zeng, ${ }^{\text {a }}$ Xiongyao Li, ${ }^{\text {a,c }}$ \\ Jianzhong Liu, ${ }^{\text {a,c }}$ and Yanxue $\mathrm{Wu}^{\mathrm{e}}$ \\ ${ }^{a}$ Center for Lunar and Planetary Sciences, Institute of Geochemistry, Chinese Academy of Sciences, Guiyang 550081, P.R. China \\ ${ }^{\text {b }}$ State Key Laboratory of Ore Deposit Geochemistry, Institute of Geochemistry, Chinese Academy of Sciences, Guiyang 550081, P.R. China \\ ${ }^{\mathrm{c}}$ Center for Excellence in Comparative Planetology, Chinese Academy of Sciences, Hefei 230026, P.R. China \\ ${ }^{d}$ College of Earth and Planetary Sciences, University of Chinese Academy of Sciences, Beijing 100049, P.R. China \\ ${ }^{\mathrm{e}}$ Guangdong University of Technology, Guangzhou 510006, P.R. China
}

Received: December 01, 2021; Revised: January 30, 2022; Accepted: January 30, 2022; Available online: January 30, 2022.

DOI: $10.46770 / A S .2022 .014$

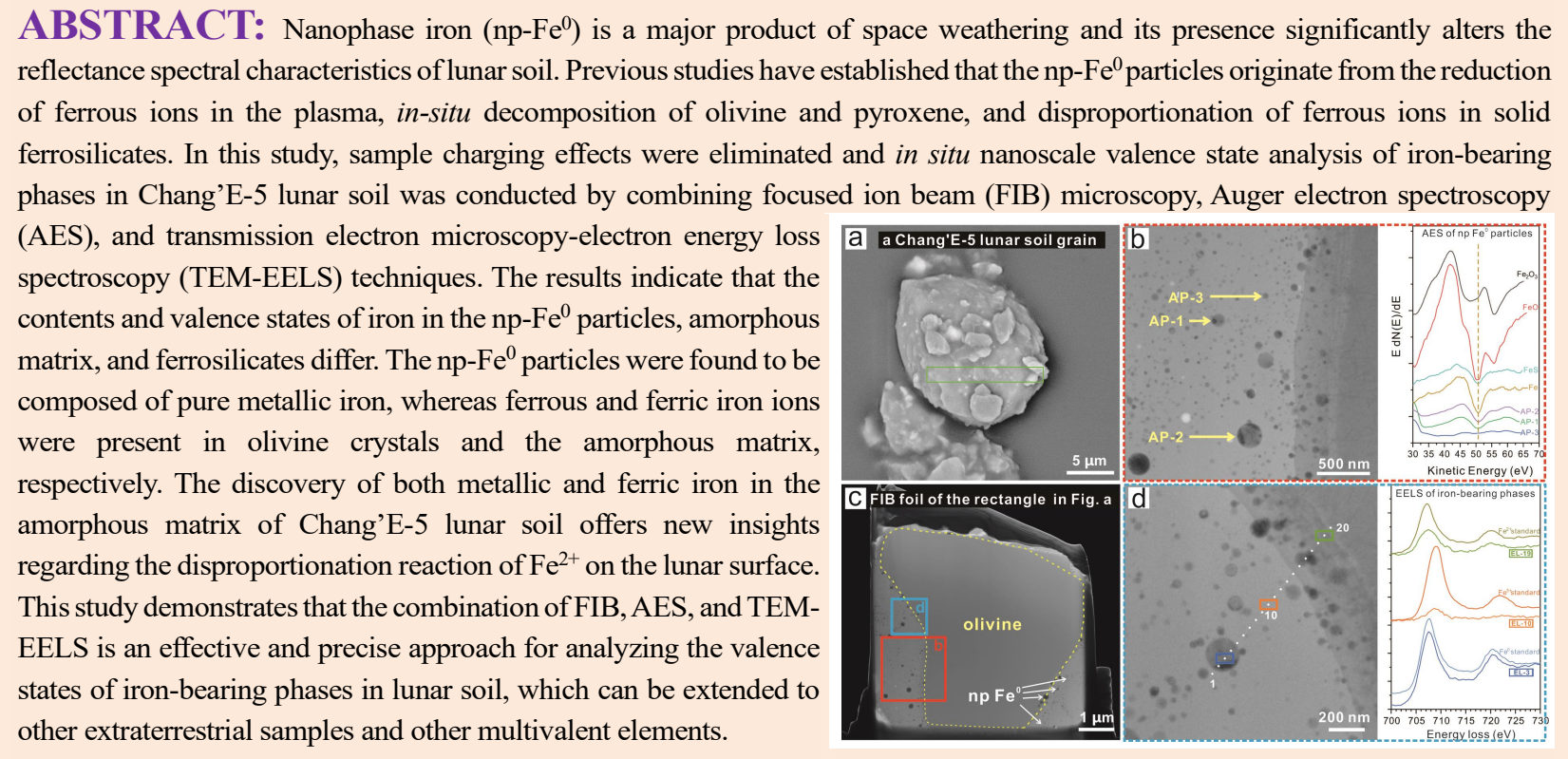

\section{INTRODUCTION}

Chang'E-5 (CE-5) successfully returned $1731 \mathrm{~g}$ of lunar soil to Earth on December 17, 2020, representing the only lunar return sample since approximately half a century. Iron is a multivalent element and the main metallic component of the lunar soil. The oxidation state of iron is a proxy for the redox environment, which is intimately linked to internal and external geological processes, such as basalt behavior, space weathering, and impact-induced modification, influencing the formation and evolution of the lunar soil.

Oxygen fugacity on the moon is considered to be at or below the iron-wüstite buffer. ${ }^{1}$ Therefore, Ferric iron $\left(\mathrm{Fe}^{3+}\right)$ rarely exists in lunar soil except in small amounts in magnetite, limonite, or associated with nanophase iron $\left(\mathrm{np}-\mathrm{Fe}^{0}\right)$ particles in amorphous 
rims and agglutinate glasses. This indicates the possibility of substantial local oxidation due to volcanic or impact-related degassing. ${ }^{2-6}$ During magmatic processes, the ferrous iron $\left(\mathrm{Fe}^{2+}\right)$ along with $\mathrm{Mg}^{2+}, \mathrm{Si}^{4+}, \mathrm{Ti}^{4+}, \mathrm{Cr}^{4+}$, and $\mathrm{O}^{2-}$ ions in lunar soil are incorporated into silicate minerals, such as olivine and pyroxene, and into oxides, such as ilmenite and chromite.

Previous investigations of the Apollo and Luna samples indicated that amorphous rims and agglutinate glasses contain approximately $0.7 \mathrm{wt} . \% \mathrm{np}-\mathrm{Fe}^{0}$ in the highlands and $1.0 \mathrm{wt} . \%$ in the mature mare lunar soil. ${ }^{7}$ The presence of np- $\mathrm{Fe}^{0}$ can not only notably alter the reflectance, absorption intensity, and continuum slope of the UV-VIS-NIR spectra of the lunar soil, but also has an appreciable impact on both the accurate interpretation of reflectance spectral remote sensing data and elucidation of lunar soil formation and evolution. ${ }^{8,9}$ Previous studies of Apollo lunar soil and lunar meteorites indicated that the formation of np- $\mathrm{Fe}^{0}$ is related to vapor deposition caused by micrometeorite impacts and the sputtered deposition and implantation of the solar wind. ${ }^{10-14}$ Although they have never been identified in lunar samples, other types of $\mathrm{np}-\mathrm{Fe}^{0}$ formation mechanisms, such as in situ reduction and disproportionation reactions, have also been detected in various types of meteorites. ${ }^{15-18}$ These results have important implications for understanding the various mechanisms of iron genesis in CE-5 lunar soil. Long-term space weathering alterations could improve the maturity of lunar soil and increase its np-Fe content. However, it can also erase characteristics of the initial stages of lunar soil formation and evolution, making it difficult to study the origin of $\mathrm{np}-\mathrm{Fe}^{0}$ in lunar soils. The short space exposure history of CE-5 lunar soil indicated that it endured relatively modest space weathering alterations. ${ }^{19}$ This provides the necessary conditions for preserving information regarding the initial evolutionary stage of lunar soil.

The iron valence states in lunar soil have been detected using Xray photoelectron spectroscopy, ${ }^{20}$ Mössbauer spectroscopy, ${ }^{21}$ ferromagnetic resonance, ${ }^{22,23}$ X-ray absorption near edge structure, ${ }^{24}$ and transmission electron microscope-electron energy loss spectroscopy (TEM-EELS). ${ }^{2,5,25}$ Herein, the valence states of iron in individual CE-5 lunar soil grains were investigated in situ using TEM-EELS, owing to the beneficial atomic spatial resolution, micro-destruction, and non-contamination features of this technique. Auger electron spectroscopy (AES) was also applied owing to its favorable surface sensitivity and the capacity to identify metallic iron oxidation. ${ }^{26,27}$

This study focuses on determining the valence states of nanophase iron-rich particles produced by the reduction of $\mathrm{Fe}^{2+}$ in silicates and by vapor deposition during space weathering or impact events, and that in the iron-bearing matrix and minerals in the CE-5 lunar soil. Auger electron spectroscopy is sensitive to sample surfaces of thickness within $10 \mathrm{~nm}$ and used to detect the oxidation and contamination characteristics of lunar soil grains in the terrestrial environment during transport, mounting, and analysis. The distribution of np- $\mathrm{Fe}^{0}$ in the CE-5 lunar soil was also determined to understand its occurrence characteristics better. The charging effects of the CE-5 lunar soil grains during AES analysis were resolved by using ultra-thin foils extracted by focused ion beam (FIB) technology, which is also appropriate for TEM-EELS. The nanoscale occurrence characteristics of np- $\mathrm{Fe}^{0}, \mathrm{Fe}^{2+}$, and $\mathrm{Fe}^{3+}$ in the CE-5 lunar soil were also investigated using EELS coupled with a Cs-corrected TEM. The results provide an important basis for determining the $\mathrm{np}-\mathrm{Fe}^{0}$ formation mechanism and the modification characteristics of iron throughout the formation and evolution of the lunar soil.

\section{EXPERIMENTAL}

Samples. Two grains were selected from CE-5 lunar soil (CE5C0400YJFM00505) allocated by the China National Space Administration. The lunar soil was collected using a shovel attached to the robotic arm of the CE-5 lander and separated into individual packages in an ultraclean room at the extraterrestrial sample curation center of the National Astronomical Observatories, Chinese Academy of Sciences (CAS). Several lunar soil grains smaller than $50 \mu \mathrm{m}$ were dispersed on aluminum double-sided tape, the other side of which was placed on a glass slide in a glove box filled with argon at the Institute of Geochemistry, CAS. The surface was then coated with a gold layer to prevent charging.

Instrumentation and operating conditions. Scanning electron microscopy images and FIB cross-sections were obtained using an FEI Scios Dual-beam system at the Institute of Geochemistry, CAS. High-resolution back-scattered electron images were obtained at an accelerating voltage of $15 \mathrm{kV}$ and a working distance of $7 \mathrm{~mm}$. The ultrathin foils were prepared using FIB according to the Wirth method ${ }^{28}$. The FIB foils were characterized in sequence using a field-emission scanning transmission electron microscope (FE-STEM), scanning Auger nanoprobe, and TEMEELS.

The nanoscale composition and structure of the FIB foils were characterized using an FEI Talos F200X FE-STEM operating at $200 \mathrm{kV}$ at the Suzhou Institute of Nano-tech and Nano-bionics, CAS. The chemical composition of the samples was analyzed using an energy-dispersive X-ray spectroscopy (EDS) detector on the STEM instrument.

The valence states and distribution of the nanophase iron-rich particles in the lunar soil were analyzed using a PHI 700 scanning Auger microprobe and a PHI 710 scanning Auger nanoprobe (Tsinghua University), the vacuum chamber of which was under a base pressure of $<8.0 \times 10^{-9}$ Torr. The accelerating voltage of the 
electron gun was set to $10 \mathrm{kV}$, and beam currents of 10,5 , and 1 $\mathrm{nA}$ were tested. The optimal signal intensity and spatial resolution were achieved under a current of $5 \mathrm{nA}$. The FIB foils were first cleaned by applying $1 \mathrm{keV} \mathrm{Ar}^{+}$beam sputtering across an area of $1 \mathrm{~mm}^{2}$ for 1 to $3 \mathrm{~min}$, and the ion incident angle was approximately $30^{\circ}$ to the sample surface. The sputtering efficiency of the $\mathrm{Ar}^{+}$ beam under such conditions was approximately $3 \mathrm{~nm} / \mathrm{min}$. Then, the foils were analyzed using a scanning Auger micro/nanoprobe. Iron and troilite in ordinary chondrite (GRV051874, L6) were prepared as $\mathrm{Fe}^{0}$ and $\mathrm{Fe}^{2+}$ references, respectively. The AES results of wüstite and hematite from Yao et al. ${ }^{27}$ were used to investigate the valence states of iron in the Fe-rich nanophase particles. The AES mapping was performed with a peak position at $50.8 \mathrm{eV}$ and a background of $55.0 \mathrm{eV}$.

TEM-EELS was performed using a Gatan GIF Quantum ER system Model 965 parallel EELS spectrometer attached to a Hitachi HF5000 TEM housed at the Shanghai Institute of Ceramics, CAS. The accelerating voltage was $200 \mathrm{kV}$, the energy resolution at the zero-loss peak was $0.5-0.7 \mathrm{eV}$ FWHM, and spectra were acquired in DualEELS mode using a probe current of $100 \mathrm{pA}$. The acquisition times were $10 \mathrm{~s}$ and $18 \mathrm{~s}$ for the point analysis and line scan, respectively. The $\mathrm{Fe}^{0}$ and $\mathrm{Fe}^{2+}$ references were the same as those used in the AES analysis, and terrestrial hematite was prepared as a $\mathrm{Fe}^{3+}$ reference to determine the valence states of iron in both the Fe-rich nanophase particles and the matrix.

\section{RESULTS AND DISCUSSION}

AES is a highly sensitive surface detection method because only Auger electrons from within a dozen atomic monolayers on the outermost surface are emitted. Therefore, the charging effects are the main drawbacks limiting the AES applicability in the analysis of insulating geological materials. Coating the sample surface with carbon or other conductive layers is unfeasible because the AES signal of only the conductive material is obtained in this case. In this study, ultrathin FIB foils with a thickness of $<100 \mathrm{~nm}$ were used for the first time for the AES analysis of geological samples, effectively overcoming the surface charging effect because the electron beam current was mostly transmitted and finally transferred to the conductive sample holder through the thin sample slice.

The FIB sampling and AES point analysis sites are shown in Fig. 1. Figure 2 shows the AES spectra collected at various points in Fig. 1. The survey spectra of AS-1/2/3 were used to obtain information regarding the sample surface cleanliness and chemical composition of the various phases. $\mathrm{The}^{+} \mathrm{Ar}^{+}$beam sputtering produces an approximately clean surface, but small amounts of carbon and oxygen remain. Because focusing the $\mathrm{Ar}^{+}$beam on the sub-micro scale is difficult and the sputtering area is $1 \mathrm{~mm}^{2}$,
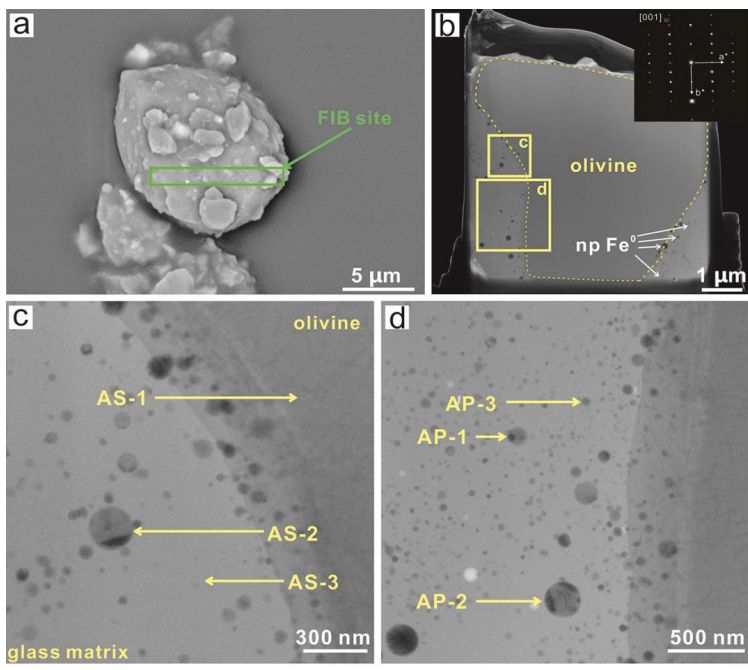

Fig. 1 Focused ion beam (FIB) sampling and AES point analysis sites. (a) Back-scattered electron image of CE5C0400YJFM00505-G1. The green rectangle indicates the FIB site. (b) Dark field image of the FIB foil. The fast Fourier transform of the mineral in the middle shows lattice spacings that are consistent with olivine, surrounded by a glass matrix containing np$\mathrm{Fe}^{0}$ particles. (c), (d) Show the analysis points of the AES survey and $\mathrm{Fe}_{\mathrm{MNN}}$ AES, respectively.

significantly larger than that of the FIB foil, some surrounding materials, such as the carbon double-sided adhesive and FIB copper mesh, may redeposit on the foil surface. However, the data in Figs. $2 b$ and $2 c$ show that Fe, being the focus of this study, is minimally affected. The AES survey data show that the FIB foil predominantly contains olivine, $\mathrm{np}-\mathrm{Fe}^{0}$, and a silicic matrix.

The differential and integral Auger line shapes of the references are shown in Figs. 2b and 2c, respectively. The peak positions in the differential spectrum are readily identified, and are useful for analyzing the oxidation of iron. ${ }^{26,27}$ Therefore, in this study, the differential spectra were utilized for identifying the iron oxidation state. The differential spectrum indicated that the AES spectra of the metallic iron and troilite are comparable, both contain only one main FemnN peak at $50.8 \mathrm{eV}$. On the other hand, the spectra of wüstite and hematite contain two FemNN peaks, one at $55.8 \mathrm{eV}$ in both cases, and at 48.0 and $50.0 \mathrm{eV}$, respectively. The AES point analysis data indicated that particles AP-1 and AP-2 are likely to be $\mathrm{Fe}$ or $\mathrm{FeS}$, rather than $\mathrm{FeO}$ and $\mathrm{Fe}_{2} \mathrm{O}_{3}$, and the survey data indicated that the particles were sulfur/nickel-free. Thus, these particles were verified to be pure metallic iron. The Fe MNN peak in the spectrum of the AP-3 particle was too weak to be identified, which indicates that AES is not suitable for analyzing particles smaller than $70 \mathrm{~nm}$ (diameter of AP-3 Fe particles). The surface distribution of $\mathrm{Fe}^{0}$ in CE5C0400YJFM00505-G2 was determined based on the AES mapping (Fig. 3), whose resolution reached the nanoscale and was comparable to that of the EDS mapping using TEM. In addition, the AES mapping contains valence state information and the surface sensitivity is higher than that of EDS mapping. 

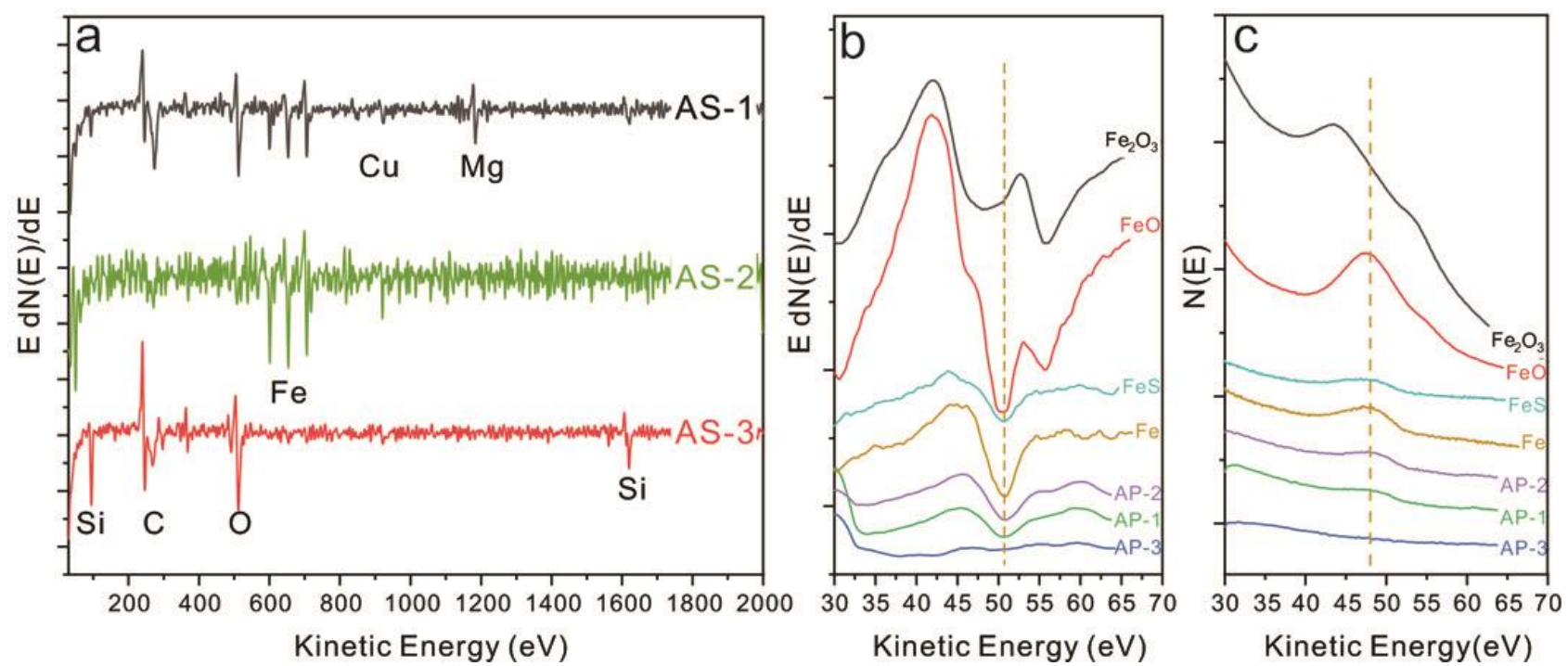

Fig. 2 (a) AES survey of the various phases in the FIB slice of CE5C0400YJFM00505-G1. (b) and (c) $\mathrm{Fe}_{\mathrm{MNN}}$ differential and integral Auger line shapes of the standard references and np- $\mathrm{Fe}^{0}$ in the glass matrix, respectively.
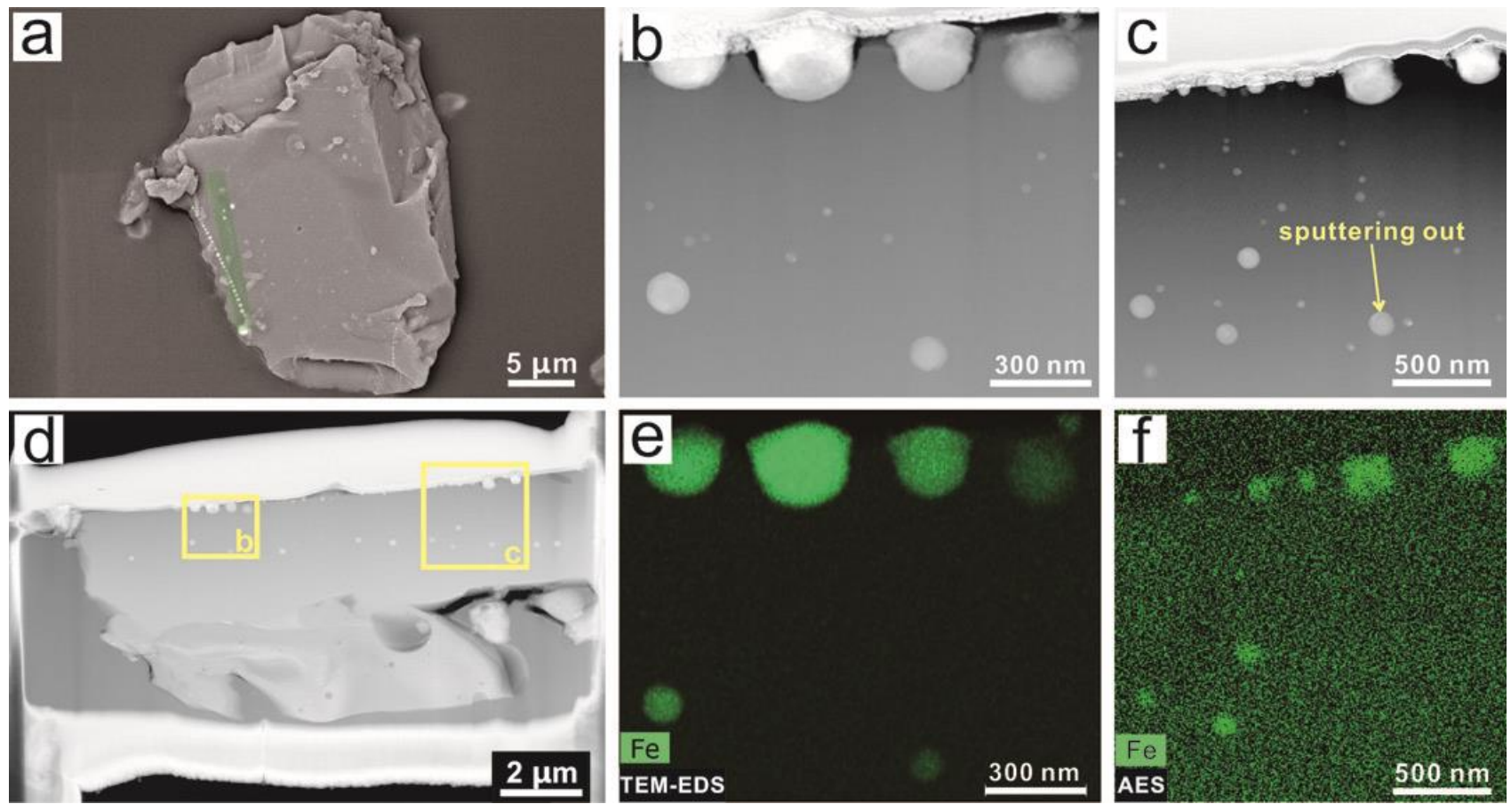

Fig. 3 (a) Back-scattered electron image of CE5C0400YJFM00505-G2. The green rectangle shows the FIB site. (b) High-angle annular dark field (HAADF) image of the TEM-EDS mapping area. (c) HAADF image of the AES mapping area. (d) HAADF image of the FIB foil extracted from CE5C0400YJFM00505-G2. (e) TEM-EDS map of iron. (f) AES map of Fe ${ }^{0}$.

Note that the line shape and peak position in the AES spectra are influenced by the element's valence state and the atom's chemical environment in the molecular structure. This implies that iron with the same valence state exhibit varying AES characteristics depending on the compound it comprises (see the AES spectra of $\mathrm{FeO}$ and $\mathrm{FeS}$ in Fig. 2). This is a limitation on investigating the valence states of iron using AES. However, the AES line shapes and peak positions of metallic iron and hematite differ substantially, thus, readily distinguished using this method. In addition to its favorable surface sensitivity, AES is still a powerful technique for detecting the surface oxidation of $n p-\mathrm{Fe}^{0}$ particles larger than $70 \mathrm{~nm}$, identifying the existence of nonsample components on the sample surface, and determining whether contamination or oxidation occurred during sample preparation, transport, or storage. Therefore, it provides a foundation for the subsequent analysis of lunar soil and other 

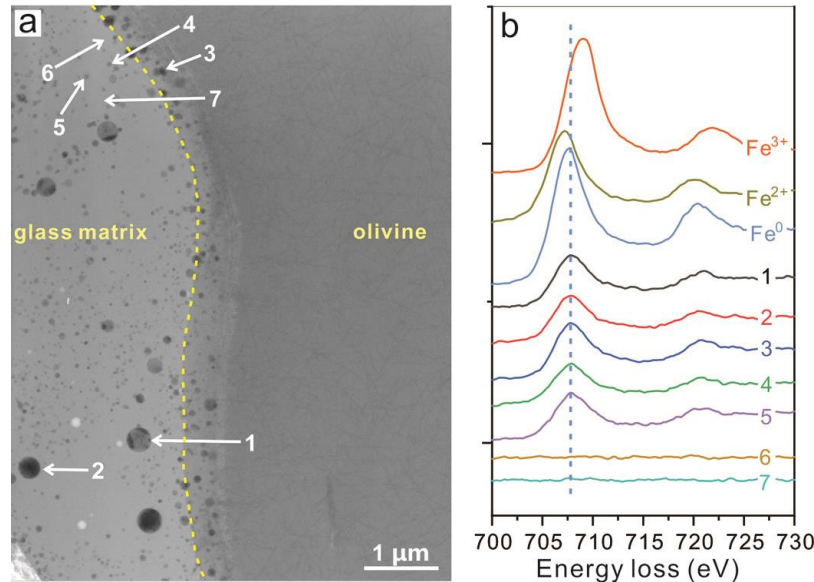

Fig. 4 (a) TEM-EELS point-analysis positions in FIB foil extracted from CE5C0400YJFM00505-G1. (b) Fe L 3,2 TEM-EELS data for the standard references and points shown in (a).

extraterrestrial samples. Furthermore, if the chemical compositions of other iron-bearing materials are determined, the iron valence states of the components can be identified using appropriate standard references.

The same FIB foil extracted from CE5C0400YJFM00505-G1 for the AES analysis was also analyzed using TEM-EELS. The TEM-EELS point and line analysis results are shown in Figs. 4 and 5, respectively. The peak intensity of the Fe TEM-EELS line scan was stronger than that of the point analysis owing to the longer acquisition time of the former. The $\mathrm{Fe} \mathrm{L}_{3,2}$ EELS data for the standard references indicate that $\mathrm{Fe}^{0}, \mathrm{Fe}^{2+}$, and $\mathrm{Fe}^{3+}$ have distinct edge shapes. $\mathrm{Fe}^{0}$ and $\mathrm{Fe}^{3+}$ exhibit notably different $\mathrm{L}_{3}$ peak positions at $707.7 \mathrm{eV}$ and $709.0 \mathrm{eV}$, respectively, and the $\mathrm{L}_{3}$ peak position of $\mathrm{Fe}^{2+}$ shifts slightly to $707.2 \mathrm{eV}$. The valence states of iron can be determined by comparing the peak positions and line shapes with those of the standard references. The TEM-EELS results of seven points, EP-1 (same analysis point as in the AES analysis) to EP-7, were used to determine the valence states of the nanophase iron-rich particles and the matrix. These results are consistent with both the AES results and those of previous studies. EP-1 and other points detected on the nanophase iron-rich particles were also metallic iron. The TEM-EELS point analysis of the matrix indicated the absence of iron in the analyzed points (EP-6 and EP-7), indicating that the Fe content in the matrix is inhomogeneous.

A TEM-EELS line scan through the $n p-\mathrm{Fe}^{0}$ particles, matrix, and olivine was analyzed to obtain further systematic information on the iron valence states in the sample. The analysis positions and results are shown in Fig. 5. The valence states of iron were determined by comparing the observed peak positions and line shapes with those of the three standard references. The TEMEELS signals may be influenced by the substrate in the presence of surrounding glassy materials lying above and/or below the nanoparticles in the cross-section, ${ }^{5,29}$ therefore, three TEM-EELS data points from the center of the three phases along the line are displayed along with the standard data for a more intuitive contrast. The Fe TEM-EELS line scan data from this sample indicated that the $\mathrm{np}-\mathrm{Fe}^{0}$ particles were metallic, while the matrix between them and olivine contained $\mathrm{Fe}^{3+}$, and the iron in olivine existed as $\mathrm{Fe}^{2+}$. The discovery of both $\mathrm{np}-\mathrm{Fe}^{0}$ and $\mathrm{Fe}^{3+}$ in the amorphous matrix of CE-5 lunar soil provides possible clues into the disproportionation reaction of $\mathrm{Fe}^{2+}$. Meteorite impact induces the melting of lunar olivine grains and leads to the valence transition of ferrous iron in the amorphous matrix. This study provides a new insight into the possible formation mechanisms of $\mathrm{np}-\mathrm{Fe}^{0}$ in lunar soils.

EELS equipped with a Cs-corrected TEM provides atomiclevel spatial resolution, and the peak positions and curve shapes of the iron TEM-EELS data differ considerably among the various valence states. Thus, this technique is an ideal tool for the in situ qualitative valence state analysis of iron-bearing phases in lunar soil. Future studies will be focused on developing an accurate quantitative calculation method and determining whether the valence state is the only factor affecting the TEM-EELS results.

The chemical composition, iron valence state, and crystal structure of lunar soil grains can be detected using a combination of AES, TEM-EELS, TEM-EDS, and selected area electron diffraction (SAED), allowing for micro-damage, contaminationfree, and in situ micro-area analysis of CE-5 and other extraterrestrial samples. AES and TEM-EELS can be extended to detect the in situ micro-distribution and the valence states of other multivalent elements in extraterrestrial samples, such as Cr, Ti, P, and Mn.

\section{CONCLUSIONS}

A combination of AES and TEM-EELS techniques was employed to identify the valence states of nanophase iron-rich particles and other iron-bearing phases in CE-5 lunar soil grains, both of which were obtained from the same FIB cross-section. AES is a useful tool for determining the surface oxidation of the $n p-\mathrm{Fe}^{0}$ particles and the surface contamination of lunar soil by earth environments owing to its favorable surface chemical composition and valence states sensitivity within $10 \mathrm{~nm}$. The distribution of iron on the sample surface can also be detected using AES, however, it is less suitable for $\mathrm{np}-\mathrm{Fe}^{0}$ particles smaller than $70 \mathrm{~nm}$. TEM-EELS is a powerful technique for analyzing the valence states of various iron-bearing phases in the lunar soil based on its atomic-scale spatial resolution and combination with TEM-EDS and SAED. Both np- $\mathrm{Fe}^{0}$ and $\mathrm{Fe}^{3+}$ were detected in the amorphous matrix of CE-5 lunar soil, providing new insights into the possible formation mechanism of the np- $\mathrm{Fe}^{0}$ particles in lunar soil and the disproportionation reaction of $\mathrm{Fe}^{2+}$ in olivine during meteorite impaction. These techniques can be further extended to analyze other multivalent elements in extraterrestrial samples. 

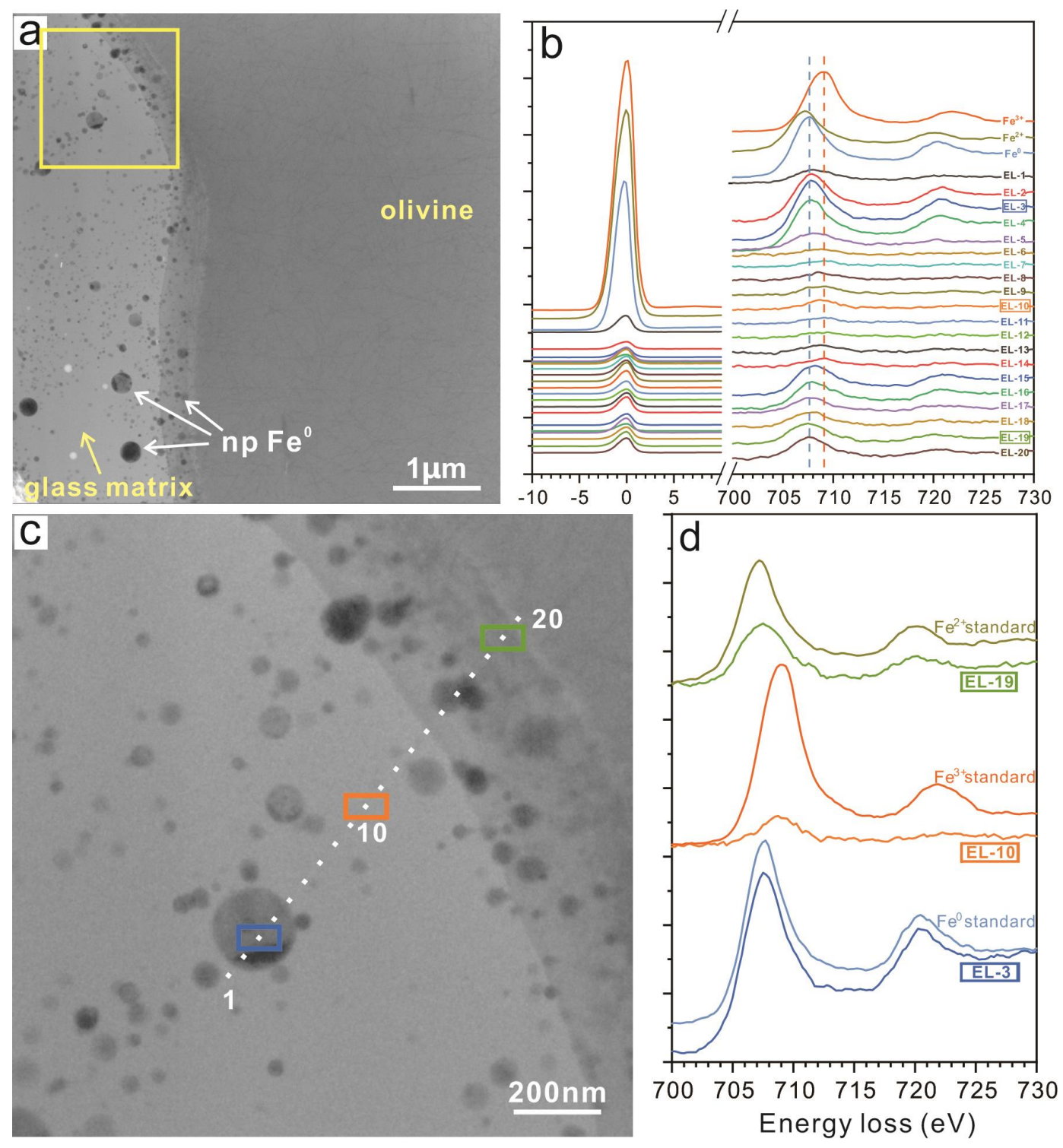

Fig. 5 (a) HAADF image of the FIB foil extracted from CE5C0400YJFM00505-G1. The yellow square shows the position of (c). (b) Fe L $\mathrm{L}_{3,2}$ TEM-EELS data of the standard references and line scan points with the aligned zero-loss peak. (c) The line analyzed by TEM-EELS and associated analysis points. (d) Three TEM-EELS results from the center of the three phases in the line along with the standard data.

\section{AUTHOR INFORMATION}

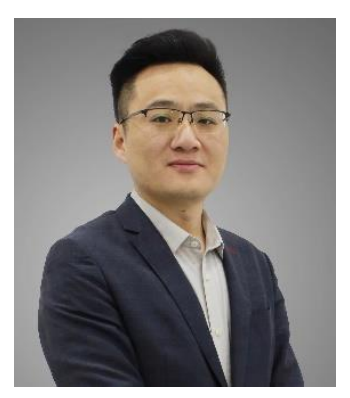

Yang $\mathbf{L i}$ is an associate professor of planetary science at the Institute of Geochemistry, Chinese Academy of Sciences (IGCAS) in Guiyang, China. $\mathrm{He}$ completed his Ph.D. in cosmochemistry from the University of Chinese Academy of Sciences in 2013. His early research focused on the space weathering processes on the moon, especially solar wind implantation and micrometeorites bombardment. His recent research interests have expanded to the formation and evolution of lunar soil as well as surface materials of asteroids, in-situ space resources utilization, high-precision FIB/TEM/EELS/SIMS microanalyses, and their applications to Planetary and Earth science. He developed a series of space environment simulation devices and experimental methods. Currently, he is the vice director of the Center for Lunar and Planetary Sciences, IGCAS. $\mathrm{He}$ is also a member of the Youth Innovation Promotion Association, CAS, and the committee of Nanogeoscience in the Geological Society of China. He has published over 30 peer-reviewed scientific papers in ISI-indexed journals.

\section{Corresponding Author}

*Y. Li

Email address: liyang@mail.gyig.ac.cn 


\section{Notes}

The authors declare no competing financial interest.

\section{ACKNOWLEDGMENTS}

The authors would like to thank the funding supported from the Strategic Priority Research Program of the Chinese Academy of Sciences (XDB 41000000), the National Natural Science Foundation of China (41931077), the Technical Advanced Research Project of Civil Space (D020201), the Youth Innovation Promotion Association CAS (2020395), the Key Research Program of Frontier Sciences, CAS (ZDBS-SSW-JSC007-10 and QYZDY-SSW-DQC028) Guangxi Scientific Base and Talent Special Projects (AD1850007).

\section{REFERENCES}

1. B. L. Jolliff, M. A. Wieczorek, C. K. Shearer, and C.R. Neal, New Views of the Moon. Mineralogical Society of America, 2006.

2. M. S. Thompson, T. J. Zega, P. Becerra, J. T. Keane, and S. Byrne, Meteorit. Planet. Sci., 2016, 51, 1082-1095. https://doi.org/10.1111/maps.12646

3. K. H. Joy, C. Visscher, M. E. Zolensky, T. Mikouchi, K. Hagiya, K. Ohsumi, and D. A. Kring, Meteorit. Planet. Sci., 2015, 50, 1157-1172. https://doi.org/10.1111/maps.12462

4. M. C. McCanta, M. D. Dyar, M. J. Rutherford, A. Lanzirotti, S. R. Sutton, and B. J. Thomson, Icarus, 2017, 285, 95-102. https://doi.org/10.1016/j.icarus.2016.12.029

5. K. D. Burgess and R. M. Stroud, J. Geophys. Res. Planets, 2018, 123, 2022-2037. https://doi.org/10.1029/2018je005537

6. S. Li, P. G. Luceyl, A. A. Fraeman, A. R. Poppe, V. Z. Sung, D. M. Hurley, and P. H. Schultz, Sci. Adv., 2020, 6, aba1940. https://doi.org/10.1126/sciadv.aba1940

7. R. Morris, Proc. Lunar Planet. Sci., 1980, 11, 1697-1712.

8. S. Sasaki, K. Nakamura, Y. Hamabe, E. Kurahashi, and T. Hiroi, Nature, 2001, 410, 555-557. https://doi.org/10.1038/35069013

9. C. M. Pieters and S. K. Noble, J. Geophys. Res. Planets, 2016, 121, 1865-1884. https://doi.org/10.1002/2016je005128

10. L. P. Keller and D. S. McKay, Science, 1993, 261, 1305-1307. https://doi.org/10.1126/science.261.5126.1305

11. L. P. Keller and D. S. McKay, Geochim. Cosmochim. Acta., 1997, 61, 2331-2341. https://doi.org/10.1016/S0016-7037(97)00085-9
12. S. K. Noble, L. P. Keller, and C. M. Pieters, Meteorit. Planet. Sci., 2005, 40, 397-408. https://doi.org/10.1111/j.19455100.2005.tb00390.x

13. M. Anand, L. A. Taylor, M. A. Nazarov, J. Shu, H. K. Mao and R. J. Hemley, P. Natl. Acad. Sci. USA, 2004, 101, 6847-6851. https://doi.org/10.1073/pnas.0401565101

14. K. Wang, F. Moynier, F. A. Podosek and J. Foriel, Earth Planet. Sci. Lett., 2012, 337, 17-24. https://doi.org/10.1016/j.epsl.2012.05.021

15. B. van de Moortele, B. Reynard, P. Rochette, M. Jackson, P. Beck, P. Gillet, P. F. McMillan, and C. A. McCammon, Earth Planet. Sci. Lett., 2007, 262, 37-49. https://doi.org/10.1016/j.eps1.2007.07.002

16. Z. Guo, Y. Li, S. Liu, H. F. Xu, Z. D. Xie, S. J. Li, X. Y. Li, Y. T. Lin, I. M. Coulson, and M. M. Zhang, Geochim. Cosmochim. Acta, 2020, 272, 276-286. https://doi.org/10.1016/j.gca.2019.10.036

17. Z. Guo, Y. Li, H. Y. Chen, M. M. Zhang, Y. X. Wu, B. Hui, S. Liu, I. M. Coulson, S. J. Li, X. Y. Li, J. Z. Liu, and Z. Y. Ouyang, J. Geophys. Res. Planets, 2021, 126, e2020JE006816. https://doi.org/10.1029/2020je006816

18. L. Bindi, S. H. Shim, T. G. Sharp, and X. D. Xie, Sci. Adv., 2020, 6, eaay7893. https://doi.org/10.1126/sciadv.aay7893

19. Y. Qian, L. Xiao, J. W. Head, C. Wöhler, R. Bugiolacchi, T. Wilhelm, S. Althoff, B. Ye, Q. He, Y. Yuan and S. Zhao, Geophys. Res. Lett., 2021, 48, e2021GL095341. https://doi.org/10.1029/2021GL095341

20. C. A. Dukes and R. A. Baragiola, Icarus, 2015, 255, 51-57. https://doi.org/10.1016/j.icarus.2014.11.032

21. R. V. Morris, G. Klingelhofer, R. L. Korotev, and T. D. Shelfer, Hyperfine Interact., 1998, 117, 405-432. https://doi.org/10.1023/a:1012699511670

22. F. D. Tsay and S. L. Manatt, Geochim. Cosmochim. Acta, 1971, 35, 865-875. https://doi.org/10.1016/0016-7037(71)90001-9

23. R. Morris and W. Gose, Proc. Lunar Planet. Sci.. 1976, 7, 1-11.

24. L. Hicks, J. Bridges, T. Noguchi, and J. Piercy, in European Planetary Science Congress. 2020, EPSC2020-715.

25. K. Burgess and R. Stroud, Micros. Today, 2017, 25, 32-39. https://doi.org/10.1017/S1551929517000372

26. A. G. Sault, Appl. Surf. Sci., 1994, 74, 249-262. https://doi.org/10.1016/0169-4332(94)90006-X

27. W. Q. Yao, Y. X. Wu, L. P. Yang, X. Y. Li, T. G. Xu, Z. P. Li, Y. J. Wang, Z. Y. Ouyang, Y.F. Zhu, State Administration for Market Regulation and Standardization Administration of China, GB/T 36533-2018 2018.

28. R. Wirth, Chem. Geol., 2009, 261, 217-229. https://doi.org/10.1016/j.chemgeo.2008.05.019

29. L. Keller and S. J. Clemett, Lunar and Planetary Science XXXII, Houston, TX, USA, 2001, 2097. 